\title{
On the degree of attention and capacity limitation in tactile processing*
}

\author{
RICHARD M. SHIFFRIN, JAMES C. CRAIG, and ELI COHEN \\ Indiana University, Bloomington, Indiana 47401
}

\begin{abstract}
Two conditions were used to evaluate Os' ability to detect near-threshold vibrotactile stimuli. In both conditions, a signal was presented at one of three skin loci, the $O$ stating which locus received the stimulus. In the simultaneous condition, there was a single observation interval during which a signal occurred at one of the three loci. In the sequential condition, there were three observation intervals presented successively at $800-\mathrm{msec}$ intervals, with a signal presented at one of the three loci during one of the three observation intervals. Each observation interval was associated with a particular locus. Theories postulating attentional control and limitations of capacity during this type of vibrotactile information processing predict performance to be inferior in the simultaneous condition, since that condition requires sharing of attention among the three possible sources of tactile stimulation. The data showed performance to be identical in the two conditions. This result not only disconfirms the single-channel model proposed by Franzen, Markowitz, and Swets (1970), but also argues for an unlimited-capacity nonattention model of tactile perceptual processing. The results are in accord with similar findings for visual processing by Shiffrin and Gardner (1972).
\end{abstract}

One of the most basic questions in information processing concerns limitations of capacity and attentional control during perceptual encoding. Figure 1 presents three commonly proposed models of sensory processing. The top panel illustrates a single-channel model, in which information can enter the system from only a single source at any one moment. It is assumed in this model that attention switches rapidly among various possible sensory channels. Broadbent (1958) proposed a model similar to this in the context of dichotic listening experiments. Estes and Taylor $(1964,1966)$ proposed a quantitative model of this general type for visual processing. A less extreme attentional model is shown in the middle panel of Fig. 1. This attenuation model assumes total processing capacity to be limited, but allows simultaneous processing from many channels at once. The O's attention controls the attenuation of each channel, and hence the relative amounts processed in various channels. Treisman (1969), Moray (1970), and Neisser (1967) have proposed models of this sort based primarily on evidence from dichotic listening and other auditory experiments. Rumelhart (1970) and Norman and Rumelhart (1970) have proposed a model of this type based on experimentation in visual processing. As Neisser (1967) points out, both types of limited-capacity attentional models require a "preattentive" mechanism to direct attention to important channels. The final class of models is illustrated in the lower panel of Fig. 1. These models assume no attention during perceptual processing and, under many conditions, no limitations on capacity. They assume instead that attentional effects occur in short-term memory following the early phases

*This research was supported in part by NIMH Grant MH 12717-06 to the first author and in part by NIH Grant NS-09783 to the second author. of processing. Deutsch and Deutsch (1963) and Deutsch, Deutsch, and Lindsay (1967) have proposed such a model for auditory processing. Shiffrin and Gardner (1972), Shiffrin, Gardner, and Allmeyer (1972), and Shiffrin and Geisler (1972) have proposed this model for visual processing and for general sensory processing.

The classification of models in Fig. 1 is meant to be representative, not exhaustive. Listing and categorizing the many variants of attentional models would not be productive. Nevertheless, almost all models propose that one of the versions depicted in Fig. 1 holds at some level of processing. By processing level, we refer to the extent to which environmental sensory information is processed in a parallel independent or nonattentive manner before limitations of capacity and attention begin to operate. All workers appear to agree that attentional processes operate on limited-capacity information systems in short-term memory, or in any highly central level of processing. The present paper will concern itself with the question of attention operating at very early stages of processing during detection of near-threshold information.

The Shiffrin and Gardner (1972) and Shiffrin et al (1972) results are particularly important both because they place strict and clear limits on possible capacity limitations and attention during visual processing and because they utilized a paradigm which provides a model for the present experiments examining tactile processing. In the Shiffrin et al experiment, $O$ had to detect which of four spatial locations contained a brief visual signal. In the simultaneous condition, the four locations were observed at the same time, presumably requiring the sharing of attention among the four locations. In the sequential conditions, the two diagonals of the display were presented successively, $1 / 2 \mathrm{sec}$ apart, so that attention needed to be shared between two locations 

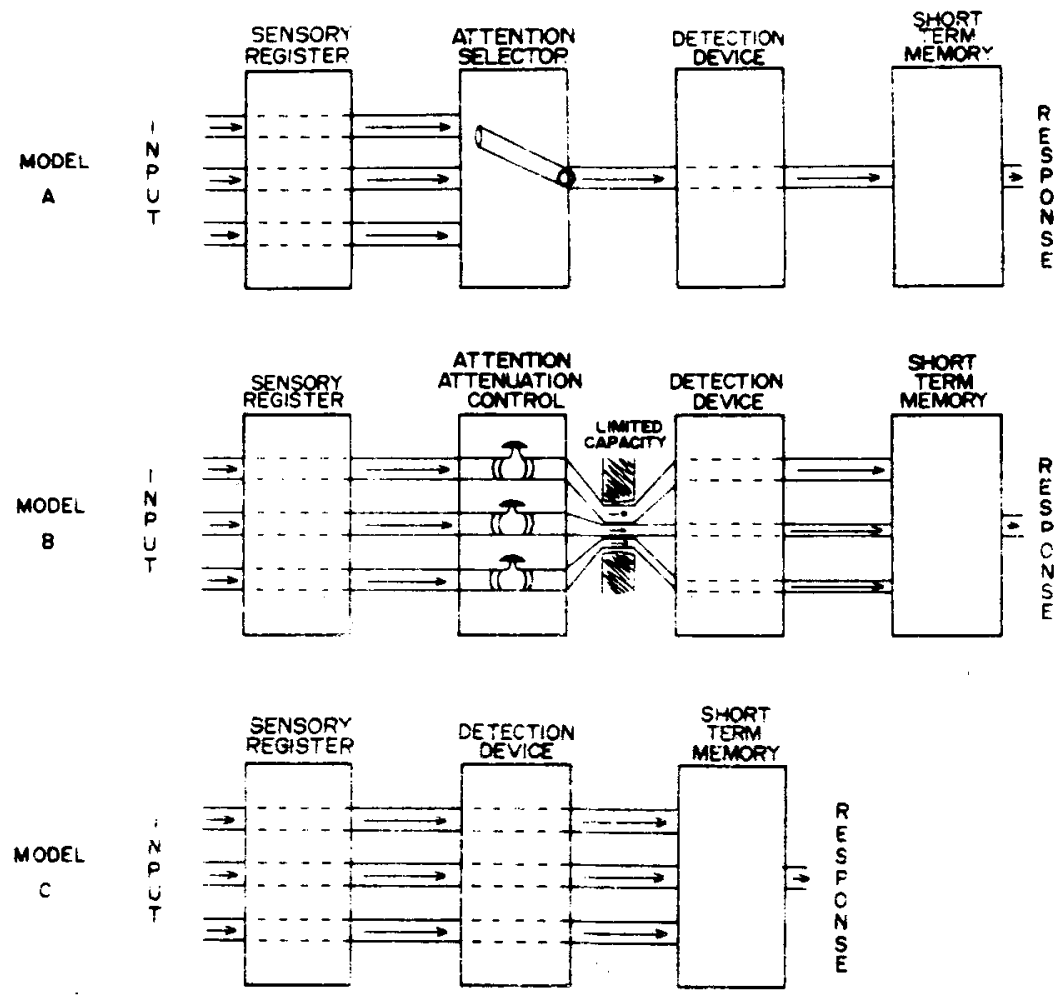

SENSORY PROCESSING MODELS
Fig. 1. Three models of attention and capacity in sensory processing. (A) Single-channel. (B) Attenuation. (C) Automatic, unlimited. only. Diagonals were presented rather than single locations in order to control for eye movements. The results demonstrated equal performance in the sequential and simultaneous conditions, thus arguing against limitations of capacity or the use of attention. This basic result was seen in a series of experiments varying the type of presentation, the materials to be detected, and the nature of distractors (if presented).

As the above references indicate, much of the testing of various models of attention has been conducted using either visual or auditory stimuli. There are, however, some data which provide evidence bearing on attentional models and which have been collected within the cutaneous modality. In a recent paper on spatial summation of vibrotactile stimuli, Franzen, Markowitz, and Swets (1970) examined the probability of detecting stimuli presented to two fingers. They used a two-interval forced-choice procedure in which one of the two temporal observation intervals contained one or two signals. When two signals were presented, they were presented simultaneously at two different sites. They reported that presenting stimuli simultaneously to two fingers did not increase the probability of detection, as compared to presenting a single stimulus to one finger. To account for their data, they proposed a single-channel model of attention for weak stimuli on the skin. The model that they propose "asserts that the $O$ can attend at any instant only to inputs to a single channel-in the present case, to a single finger [Franzen et al, 1970, p. 194]." The single-channel model is represented by $\mathrm{A}$ in Fig. 1.

In an earlier study of spatial summation using a similar paradigm, Craig (1968) reported that two vibratory stimuli presented simultaneously to two loci on the skin required less energy to be detected than presenting either stimulus by itself. This effect was obtained whether the two stimuli were on the same thigh, the fingers of the same hand, or on the fingers of the two hands. Craig indicated that this advantage for two stimuli was due to two factors: one termed "statistical summation," which is the expected probabilistic advantage associated with two independent chances at detection, and one termed "spatial summation," which is the additional advantage assumed to be the result of some physiological interaction between the two signals. To demonstrate statistical summation, in one condition of the experiment. Os received stimuli successively at two loci within the same observation interval. This condition was compared with one presenting the same stimuli simultaneously, which permits the possibility of spatial summation. In all cases. Os were informed which loci would be stimulated and whether the stimuli would be presented simultaneously or successively. A single-channel model of attention of the type proposed by Franzen et al generally predicts that performance would be superior for the successive 
condition, although, as will be shown below, such a prediction depends upon certain assumptions about detection processes. Craig's results showed that performance under the successive condition was never superior to performance under the simultaneous condition. Also, with conditions that one would predict would lead to spatial summation, viz, the same high-frequency stimuli delivered to both - sites, performance in the simultaneous condition was superior to performance in the successive condition. Craig's results are in disagreement with the results obtained by Franzen et al and are not consistent with the single-channel model of attention they propose.

It should be pointed out that the Franzen et al results are not necessarily indicative of a single-channel model. There are a variety of models proposing multiple and independent channels which do not always predict an advantage for two signals over one signal. In the two-interval forced-choice procedure used by Franzen et al (1970) and Craig (1968), let the probability of correct choice of an interval when one signal is presented in that interval be $P\left(C_{1}\right)$ and when two signals are presented in that interval be $P\left(C_{2}\right)$. Now assume that each relevant input channel has a probability $\mathrm{H}$ that a signal presented will be "detected" and a probability $F$ that a "detection," i.e., "false alarm," will occur when no signal was in fact present. Assume a decision rule in which $\mathrm{O}$ chooses the interval containing one or more "detected" signals if the other interval contained no "detected" signals. Otherwise, 0 will guess at random between the intervals. Then, for either one or two signals presented, the probability of a correct location judgment will be the sum of (1) the probability that one (or more) signals are "detected" in the correct interval and that no signals are "detected" in the incorrect interval, plus (2) the probability of guessing correctly (1/2) times the probability that both intervals have at least one "detection" or that neither interval has a detection. That is,

$$
\begin{aligned}
P\left(C_{1}\right) & =H(1-F)+1 / 2[H F+(1-H)(1-F)] \\
& =1 / 2+1 / 2(H-F)
\end{aligned}
$$

and

$$
\begin{aligned}
P\left(C_{2}\right)= & {\left[1-(1-H)^{2}\right](1-F)^{2}+1 / 2\left\{(1-H)^{2}(1-F)^{2}\right.} \\
& \left.+\left[1-(1-H)^{2}\right]\left[1-(1-F)^{2}\right]\right\} \\
= & 1 / 2+(H-F)-1 / 2\left(H^{2}-F^{2}\right)
\end{aligned}
$$

Hence

$$
P\left(C_{2}\right)-P\left(C_{1}\right)=1 / 2[H-F][(1-F)-H] .
$$

Assuming that $\mathrm{H}>\mathrm{F}$.
(a) if $\mathrm{H}=1-\mathrm{F}$,
$\mathrm{P}\left(\mathrm{C}_{2}\right)-\mathrm{P}\left(\mathrm{C}_{1}\right)=0$
(b) if $\mathrm{H}<1-\mathrm{F}$,
$\mathrm{P}\left(\mathrm{C}_{2}\right)-\mathrm{P}\left(\mathrm{C}_{1}\right)>0$
(c) if $\mathrm{H}>1-\mathrm{F}$,
$\mathrm{P}\left(\mathrm{C}_{2}\right)-\mathrm{P}\left(\mathrm{C}_{1}\right)<0$

Consequently, the present model can predict no difference, an advantage for two signals over one signal or an advantage of one signal over two signals, depending only on the values of $H$ and $F$. A similar result was pointed out by Guth (1971). Based on these equations, the differing results obtained by Craig and Franzen et al could have been due to different values of $\mathrm{H}$ and $\mathrm{F}$. We do not wish to defend this particular model, but merely point out that the conclusion of Franzen et al is highly dependent on particular model assumptions. Considering this fact and considering the prior conflicting results found by Craig, it becomes clear that a better test of an attentional model is needed. The present experiment attempts to provide a straightforward, simple, and clear test of an attentional model.

Prior to presenting the experimental paradigm, we wish to discuss the type of attentional model which will be tested. The three models of Fig. 1 can be applied at many different levels of processing. Thus, Shiffrin and Gardner (1972) proposed that attentional effects are located in short-term memory and apply late in processing, after automatic perceptual processes are complete. In the present case, we propose to test whether an attentional model applies to early stages of processing. Does the ability to detect the presence of a near-threshold vibrotactile stimulus at a given spatial locus depend on the total number of loci that are being monitored simultaneously for signals? Franzen et al propose that this will be the case. However, not all attentional models would propose that detection performance should decrease as the number of monitored channels increase, especially if only a single signal is presented. Some models would not predict channel capacity to be exceeded unless multiple signals were presented and/or multiple heterogeneous judgments were to be made. Such a procedure was used by Shiffrin and Gardner (1972) to test attention in vision, with negative results. However, in the present case, we have chosen to deal with the simpler paradigm in which only a single signal is presented, but in which the number of simultaneous channels on which the signal may appear is varied. This paradigm bypasses problems associated with the capacity of short-term memory to make multiple decisions. Furthermore, the proposed procedure directly tests the Franzen et al model of attention.

In constructing our experiment, we decided to use a sequential-simultaneous comparison similar to the Shiffrin and Gardner (1972) paradigm described earlier. In the present experiment, a single vibrotactile stimulus was presented on any trial at one of three skin sites. The $O$ 's task was to identify at which site the stimulus was 
presented. In the simultaneous condition, there was a single brief time interval during which the $\mathrm{O}$ monitored all three sites for the presence of a signal. In the sequential condition, there were three successive time intervals, one of which contained a signal. The first time interval corresponded to Site 1 , the second to Site 2 , and the third to Site 3 . That is, the order of the three sites was known to $\mathrm{O}$ so that he would always receive a signal at a site that he should be "attending" at the time. The sites were chosen so as to minimize the possibility that information arising from a site might be miscoded or uncoded as to the source location. This is important, because confusions of source location will differentially affect performance in the simultaneous and sequential conditions. In the simultaneous condition, if Os detected a signal without knowing its location, they would have to guess location randomly. However, in the sequential condition, Os would be able to guess the location correctly, since temporal interval was perfectly correlated with location of signal. Thus, any confusion of the location of detected information would lead to an advantage for sequential presentation. Conversely, an observed advantage for sequential mode could be ascribed to such a confusion effect or, alternatively, to an attentional effect. Equality between sequential and simultaneous modes is more informative; such a result would be unambiguous in showing that Os were not confused concerning localization and that attention was not operative. (One might be tempted to argue that localization is more difficult than detection, and that the task of Franzen et al is not equivalent to the present one as a result. However, a finding of equality between conditions would imply no location confusions and hence imply that localization and detection were functionally equivalent in our task.)

\section{METHOD}

\section{Observers}

Os were paid undergraduate students. In the pilot experiment, one $O$ was male and one was female. In the main experiment, three Os were female and one was male.

\section{Equipment}

The programming of the stimuli was controlled by Tektronix waveform and pulse generators. The stimuli were generated by a Hewlett-Packard 200AB oscillator, the output of which went to a Grason-Stadler $829 \mathrm{C}$ electronic switch. The output of the electronic switch was led to a MacIntosh 50-W amplifier, which in turn led to one of three attenuators. The output of each attenuator went through a matching transformer to a Goodmans V-47 vibrator. A paper-tape reader randomly determined to which one of the three attenuators the output of the amplifier would be led and consequently which one of the three vibrators would be energized. In addition, on the sequential trials, the tape reader, by triggering one of three pulse generators, controlled in which observation interval the signal would appear. The three vibrators were placed on balance pans to insure a constant force of $20 \mathrm{~g}$ against the $O$ 's skin. Each vibrator was fitted with a circular contractor $6 \mathrm{~mm}$ in diam. which protruded through a fixed surround $8 \mathrm{~mm}$ in diam.

\section{Procedure}

The Os were seated comfortably at a table, with both arms extended. The vibrators were brought in contact with the Os' skin at three loci: the thenar eminence on the right hand, the left index finger $1 \mathrm{~cm}$ from the tip, and the volar surface of the forearm. Os were instructed that the experiment consisted of two kinds of trials, sequential and simultaneous. On simultaneous trials, a light would be flashed for $500 \mathrm{msec}$, signaling an observation interval. In the middle of the observation interval, a vibrotactile stimulus would be presented at one of the three loci. The $O$ was to indicate verbally at which locus the stimulus occurred. In this condition, $O$ did not know prior to the signal which loci would receive the signal and, therefore, had to monitor all three sites at once. In the sequential condition, the observation interval was flashed for $500 \mathrm{msec}$ three times, with $800 \mathrm{msec}$ between observation intervals. The $O s$ were instructed that during the first observation interval they should attend to their right hands, since if a signal was presented during this interval it would be presented at this site. Similarly, they were instructed to attend during the second interval to their left index fingers, and during the third interval to their left forearms. In this condition, a signal was presented only at a site to which the Os had been instructed to attend at that moment. A signal was presented on each trial at one of the three loci, and the Os were to indicate where the signal occurred. Os were told, for both conditions, that stimuli were equally probable at the three loci.

At the beginning of each session, which lasted approximately $1 \mathrm{~h}$, a threshold at each site was determined by a modified method of limits. The vibrotactile stimuli were $200 \mathrm{msec}$ in duration at $160 \mathrm{~Hz}$ with a rise time of $10 \mathrm{msec}$. The trials were self-paced.

Trials were grouped in pairs of 21-trial blocks, a sequential block of 21 trials and a simultaneous block of 21 trials for a total of 42 trials. This experimental design resulted in a complete replication of the experiment every 42 trials. The order of presentation of sequential and simultaneous blocks was alternated in successive 42-trial segments. Thus, blocks were presented in a repeating $A B B A$ format; the first block of the session was alternated between sequential and simultaneous. Based on the O's performance, the intensity of the vibration at a particular site was adjusted periodically throughout a session. The changes in intensity were necessary to insure that the probability correct for each site remained at approximately .65 to .70 . However, the intensity adjustments were made only between complete, successive replications of the experimental design.

Because the studies by Craig (1968) and Franzen et al (1970) were concerned with improvements in performance in detecting stimuli at two sites rather than three, a pilot study was conducted using only two sites. The sites of stimulation were the right index finger and the thenar eminence of the left hand. As in the main experiment, blocks of sequential and simultaneous were run. Twenty trials constituted a block, with every 40 trials resulting in a complete replication of the experiment. For the sequential trials, there were $250 \mathrm{msec}$ between observation intervals. Two Os were run in the pilot study. With these exceptions, the procedure and stimuli were similar to those of the main experiment.

\section{RESULTS AND DISCUSSION}

The data were first broken down by site for each $\mathrm{O}$ and condition. For all $O$ s and conditions, there were no strong biases for particular sites. Thus, for the main experiment, among all Os and conditions. the largest difference seen between the probabilities of responding to the three sites was .09 . and most of the differences 
Table 1

Probability of Correctly Identifying the Signal Locus

\begin{tabular}{lccccccccc}
\hline & \multicolumn{3}{c}{ Pilot Study } & \multicolumn{5}{c}{ Main Experiment } \\
\cline { 2 - 7 } \multicolumn{1}{c}{ Condition } & $\mathrm{O}_{1}$ & $\mathrm{O}_{2}$ & Mean & & $\mathrm{O}_{1}$ & $\mathrm{O}_{2}$ & \multicolumn{1}{c}{$\mathrm{O}_{3}$} & $\mathrm{O}_{4}$ & Mean \\
\hline Number of Observations & 1400 & 963 & & & 1071 & 1239 & 1071 & 1071 \\
Simultaneous & .77 & .76 & .765 & & .70 & .63 & .67 & .69 & .670 \\
Sequential & .78 & .74 & .760 & .73 & .65 & .62 & .70 & .675 \\
Difference & -.01 & +.02 & +.005 & -.03 & -.02 & +.05 & -.01 & -.005 \\
z Score for Difference & -.63 & +1.01 & +.40 & & -1.96 & -1.17 & +2.48 & -.52 & -0.5 \\
Level of Significance & $>.5$ & $>.3$ & $>.7$ & & .05 & $>.2$ & .014 & $>.5$ & $>.5$ \\
\hline
\end{tabular}

were much smaller. Furthermore, the probabilities of correctly identifying signals presented at various sites did not differ greatly among the sites: in this case, the largest difference was .11 , with most of the differences much smaller. For this reason, we will present and discuss only the summary results, giving the probability of correctly locating a signal averaged over the three sites. These probabilities are given in Table 1 for the pilot study and for the main experiment. The results are presented for individual Os and for the two conditions. In order to evaluate the difference between the conditions, it was assumed as a null hypothesis that the observed proportions for the simultaneous and sequential conditions were generated by an independent series of trials with a common probability correct: $P(C)$ $=$ the average of the two observed proportions. Then, the difference between the proportions is distributed, under the null hypothesis, approximately normally with mean $=\phi$ and

$$
\text { variance }=\frac{2 P(C)[1-P(C)]}{N}
$$

In the table is given the resulting $z$ score for each $O$ and the level of significance (two-tailed). As is evident from the table, no significant differences were observed in the pilot study and for two of the Os in the main experiment. A third $O$ in the main experiment showed a barely significant advantage for sequential presentation, while the last $O$ showed a sizably significant advantage for simultaneous presentation. Averaging over Os, the differences between presentation conditions are negligible for both studies. It seems reasonable to conclude on the basis of these results that there is little or no difference between sequential and simultaneous modes of presentation.

In order to insure that the results were not an artifact of the method used, additional analysis is necessary. Since the signal intensities were altered between blocks at various times, it could be argued that on half the blocks the signal intensities were so high that performance was perfect for both conditions and on half the blocks the intensities were so low that performance was at chance for both conditions. When averaged together, the various blocks could give rise to intermediate performance with no difference between conditions. To check this possibility, we should eliminate all double blocks of 42 trials (within which intensity did not vary) that have too high or too low performance. We, therefore, postulated "cutoffs" and eliminated any 42-trial block that exceeded the upper cutoff or did not reach the lower cutoff. Figure 2 presents the results for each $\mathrm{O}$ and the results averaged across Os; it plots the difference in the probability correct for the two conditions as a function of increasingly severe cutoffs. If the finding of no difference between conditions were artifactual, then a difference would be expected to appear as cutoffs were made increasingly severe. In other words, the curves in Fig. 2 would have positive slope. It is evident from inspection of the figure that the results are not artifactual. Regardless of the cutoffs used, sequential performance was equivalent to simultaneous performance.

These results indicate that at most only a small advantage of sequential over simultaneous is possible. Attention models do predict an advantage to appear, and the size of the advantage should give an indication of the amount of attention operating. The present results therefore indicate that even if attention is operating, its effects are of essentially negligible magnitude. In order to validate this conclusion, we must turn to specific models and examine the amount of advantage that those models predict under the present conditions.

We are therefore going to examine two models. The "magnitude" model assumes the $\mathrm{O}$ makes good use of available information, and it tends to maximize the predicted advantage of sequential over simultaneous presentation. The "criterion" model makes minimal use of available information, and it tends to minimize the predicted advantage of sequential over simultaneous. Therefore, the quantitative results from these two models should cover the range of results possible from most sensible models.

For each model, we will show that a "single-channel" version is not adequate to explain the data. Then we will estimate within each model the maximum amount of attention consistent with our results. We turn now to the presentation of the models.

\section{MODELS FOR THE EXPERIMENTS}

The models we consider will assume that the $O$ 


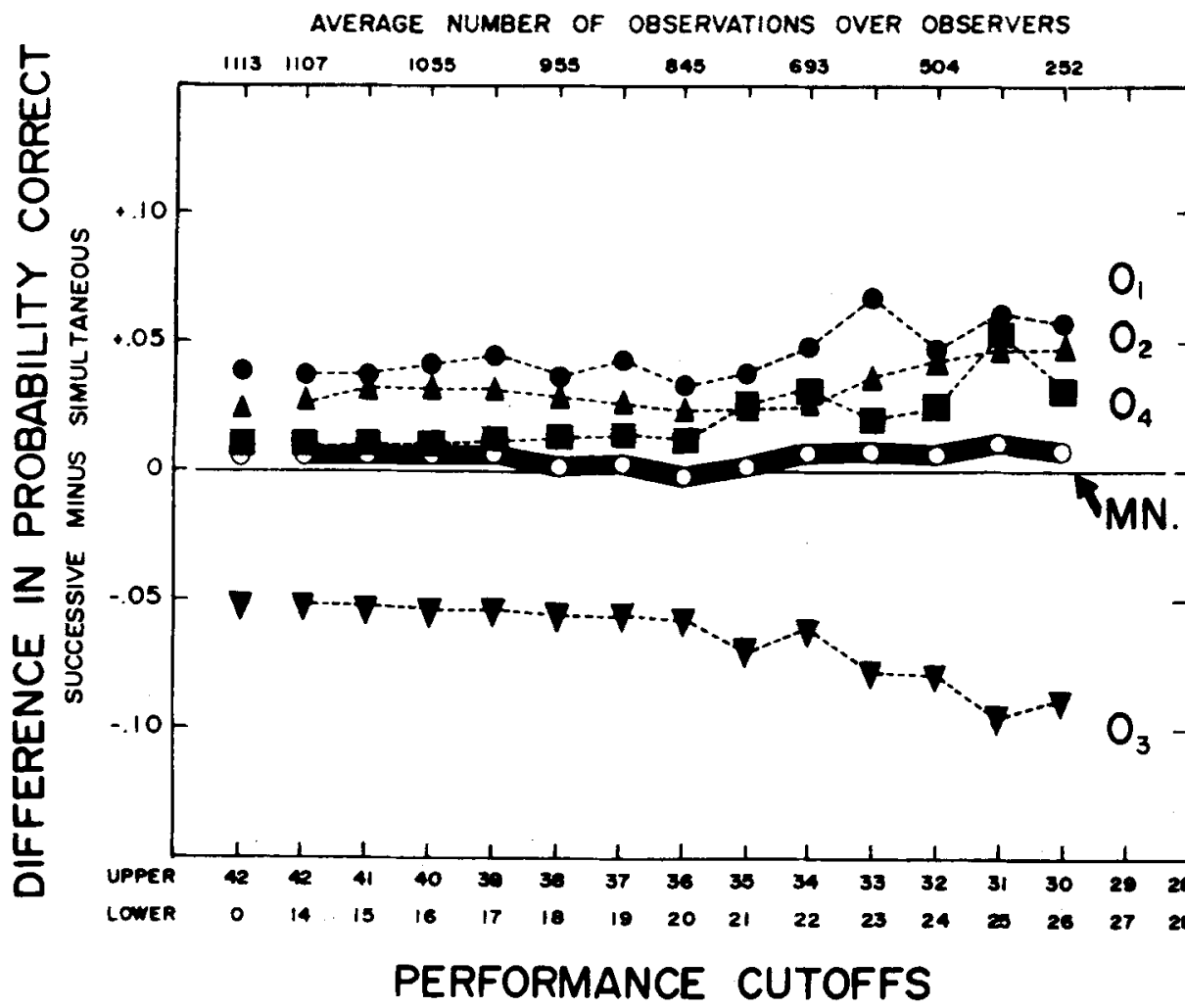

Fig. 2. Successive minus simultaneous probability correct as a function of increasingly severe performance cutof $f$. The points plotted are based on blocks of 42 trials which do not exceed the upper cutoff and do not fall below the lower cutoff in terms of number of trials correct.

attends to some proportion (possibly 1) of the various input channels. On each attended channel, the $O$ receives a sensory signal of some magnitude. The criterion model assumes that the $\mathrm{O}$ bases his decisions on those channels with sensory signal magnitudes above a criterion; this model ignores the relative magnitudes of these signals. The magnitude model assumes that the $\mathrm{O}$ bases his decision on the magnitudes of the signals perceived on the attended channets. In the following discussions, let the probability of correct location judgment for sequential presentations be denoted $P(e, n)$ and let the same probability for simultaneous presentations be denoted $P(i, n)$, where $n$ denotes the number of channels.

\section{The Criterion Model}

For each attended channel, let the probability that a perceived signal is above criterion be denoted $\alpha$ when a signal was actually presented on that channel and $\beta$ when no signal was presented on that channel.

For simultaneous presentation, assume that the $O$ always picks one channel and attends to that channel. Each other channel is attended to with probability $x$. When $\mathrm{x}=0$, we have a single-channel model similar to that of Franzen et al (1970) and related to that of Broadbent (1958). When $0<x<1$, we have an attenuation model similar to those of Rumelhart (1970). Neisser (1967), and Treisman (1969). When $x=1$, we have a nonattention model.
If one or more channels have signals above criterion, then the $O$ guesses among these randomly. If no channels have signals above criterion, then the $O$ guesses at random among the nonattended channels, or at random among all channels if there are no nonattended channels.

For sequential presentation, we assume that every channel is attended; in all other respects, the above model applies. Thus, the sequential case is identical to the simultaneous case when $\mathrm{x}=1$.

In order to see how we derive the probability of correct location judgment for this model, assume that three channels are being used. Note that in the sequential condition, all channels are attended. Hence, the probability correct in this condition will be the sum of (1) the probability that a signal is "detected" in the correct interval and that no signals are detected in the other two intervals, plus (2) the probability of detecting signals in the correct interval and in exactly one of the other two intervals times the probability of guessing the correct interval from among these two (1/2), plus (3) the probability of detecting signals in all three intervals times the probability of correctly guessing among these three $(1 / 3)$. plus (4) the probability of detecting signals in none of the three intervals times the probability of guessing correctly among these three (1/3). A similar. but simpler. derivation is used when there are only two intervals.

The derivations in the simultaneous conditions are 
somewhat more complex, because the number of attended channels can vary from one to three. Assume, again, that three channels are being used. Then the probability correct can be written as the sum of (1) the probability that three channels are attended $\left(x^{2}\right)$ times the probability correct given that three channels are attended, plus (2) the probability that two channels are attended $[2 x(1-x)]$ times the probability correct given two channels are attended, plus (3) the probability that only one channel is attended $\left[(1-x)^{2}\right]$ times the probability correct given one channel is attended. The above three conditional probabilities are easy to derive: the first has been given in the preceding paragraph. The second can be written as the sum of (1) the probability that the correct channel is not being attended (1/3) times the probability correct given the correct channel is not attended, plus (2) the probability that the correct channel is attended $(2 / 3)$ times the probability correct given the correct channel is attended. The third conditional probability can be written as the sum of (1) the probability that the correct channel is attended (1/3) times the probability correct given the correct channel is attended, plus (2) the probability that the correct channel is not attended $(2 / 3)$ times the probability correct given the correct channel is not attended. A similar but simpler derivation is used when there are only two intervals.

The resulting equations are given below. Remember that $P(j, k)$ refers to the probability correct when the condition is Type $\mathrm{j}$ and the number of channels is $k$.

$$
\begin{aligned}
\mathrm{P}(\mathrm{e}, 2) & =\alpha(1-\beta)+\alpha \beta / 2+(1-\alpha)(1-\beta) / 2 \\
& =1 / 2+1 / 2(\alpha-\beta) . \\
\mathrm{P}(\mathrm{i}, 2) & =\mathrm{xP}(\mathrm{e}, 2)+(1-\mathrm{x})[1 / 2 \alpha+1 / 2(1-\beta)] \\
& =1 / 2+1 / 2(\alpha-\beta) .
\end{aligned}
$$

Thus,

$$
P(e, 2)-P(i, 2)=0
$$

(regardless of the value of $\mathrm{x}$ ).

$$
\begin{aligned}
\mathrm{P}(\mathrm{e}, 3)= & \alpha(1-\beta)^{2}+(1 / 2)(2) \alpha \beta(1-\beta)+1 / 3 \alpha \beta^{2} \\
& +1 / 3(1-\alpha)(1-\beta)^{2} \\
= & 1 / 3+1 / 3(\alpha-\beta)(2-\beta) . \\
\mathrm{P}(\mathrm{i}, 3)= & x^{2} \mathrm{P}(\mathrm{e}, 3)+2 \mathrm{x}(1-\mathrm{x})\left\{1 / 3(1-\beta)^{2}\right. \\
& +2 / 3[\alpha(1-\beta)+1 / 2 \alpha \beta]\} \\
& +(1-x)^{2}[1 / 3 \alpha+(2 / 3)(1 / 2)(1-\beta)]
\end{aligned}
$$

$$
=1 / 3+1 / 3[\alpha-\beta]\left[1+2 x-x^{2}+\beta x(x-2)\right]
$$

Thus,

$$
P(e, 3)-P(i, 3)=1 / 3[\alpha-\beta]\left[1-\beta(1-x)^{2}-x(2-x)\right]
$$

and,

$$
P(e, 3)-P(i, 3)=1 / 3[\alpha-\beta][1-\beta], \text { if } x=0
$$

Equation 6 shows that, for the criterion model, sequential is not predicted to be superior to simultaneous, regardless of the amount of attention, if only two channels are used. On the other hand, Eqs. 9 and 10 show that sequential is superior to simultaneous when three channels are used, as long as $x<1$, which implies some attention operating, and $\beta<\alpha$, which must be true according to the definitions of $\beta$ and $\alpha$. Further discussion is deferred until the magnitude model has been presented.

\section{The Magnitude Model}

Assume that the $O$ perceives a sensory magnitude on every channel and chooses the channel with the largest magnitude. Assume that the distribution of perceived magnitudes on channels not containing a signal is normal with mean $\mu$ and variance $\sigma^{2}$; the distribution on a channel containing a signal is normal with mean $\mu+\mathrm{d}^{\prime}$, variance $\sigma^{2}$. Let $\mathrm{C}\left(\mathrm{d}^{\prime}, \mathrm{n}\right)$ denote the probability that the correct channel will be chosen (i.e., that the channel containing the signal will have the largest magnitude), where $n$ is the number of channels. Then Swets (1964) gives:

$C\left(d^{\prime}, n\right)$

$$
=1-(n-1) \int_{-\infty}^{\infty}[\operatorname{GAU}(t)]^{n-2} \mathrm{GAU}\left(\mathrm{t}-\mathrm{d}^{\prime}\right) \operatorname{gau}(\mathrm{t}) \mathrm{dt}
$$

where "GAU" is the standard normal distribution function and "gau" is the standard normal density. Swets also supplies tables for these functions.

For the simultaneous condition assume that the $O$ attends to one channel and that a signal on this channel will have a distribution with mean $\mu+d^{\prime}$; assume a signal on any other channel will have a distribution with mean $\mu+x d^{\prime}$, where $0 \leqslant x \leqslant 1$. Thus, all channels except the attended one will be attenuated to some degree.

For the sequential condition, a signal on any channel is assumed to have a distribution with mean $\mu+d^{\prime}$. For both conditions, a channel without a signal is assumed to give a distribution with mean $\mu$. All distributions are 
assumed to be normal with variance $\sigma^{2}$.

These assumptions give us:

$$
\begin{aligned}
& P(e, n)=C\left(d^{\prime}, n\right) \\
& P(i, n)=(1 / n) C\left(d^{\prime}, n\right)+\frac{n-1}{n} C\left(x d^{\prime}, n\right)
\end{aligned}
$$

Thus,

$$
P(e, n)-P(i, n)=\frac{n-1}{n}\left[C\left(d^{\prime}, n\right)-C\left(x d^{\prime}, n\right)\right]
$$

Equation 13 is the sum of (1) the probability that the signal is presented on the attended channel times the probability correct given the signal is presented on the attended channel, plus (2) the probability that the signal is presented on a nonattended channel times the probability correct given that the signal is presented on a nonattended channel.

Although it may not be obvious from cursory examination of Eq. $11, \mathrm{C}\left(\mathrm{xd}^{\prime}, \mathrm{n}\right)$ is a monotonically increasing function of $x$ (see Swets, 1964). Hence, Eq. 14 shows that sequential is always superior to simultaneous for this model when more than one channel is used.

\section{Discussion of the Models and the Results}

The criterion model is examined because it tends to minimize the differences between successive and simultaneous conditions for attentional models. The guessing strategy used in the simultaneous conditions is quite efficient for small $n$. For $n=2$, no difference between simultaneous and sequential is predicted, even for a single-channel model $(x=0$, see Eq. 6$)$. In words, the strategy of guessing the nonattended channel is as efficient as attending to that channel, if no magnitude information is utilized in decisions. This model may not be a likely alternative, but its existence rules out the use of the pilot study as a conclusive test of an attentional model. As indicated in Eqs. 9 and 10, however, an advantage for sequential is predicted for $n=3$ (if $\alpha>\beta$ and $x<1$ ). For these reasons, we terminated the pilot study $(n=2)$ and ran the main experiment with the three channels $(n=3)$.

The pilot study was quite informative, these considerations notwithstanding. First, it bridges the gap between previous studies using two channels and the main experiment which uses three channels. As a result, the main findings cannot be attributed to some special characteristic of three channels. Second, only a model which maximizes performance in the simultaneous condition and minimizes performance in the sequential condition predicts no advantage for sequential. An attentional model that assumes more intelligent decisions during the sequential conditions predicts an advantage for sequential. The magnitude model demonstrates this fact (see Eq. 14). Thus, the finding of no difference in the pilot study provides at least partial evidence against an attentional model.

The crucial test of the attentional approach occurs during the main experiment. The finding that sequential presentation had no advantage over simultaneous was in agreement with an unlimited-capacity nonattentional model and in conflict with the attentional models, as indicated in Eqs. 9, 10, and 14. First note that even the single-channel criterion model (Eq. 10) predicts a sizable advantage for sequential mode. It may be shown that if $\mathrm{P}(\mathrm{e}, 3) \cong .67$, which was observed, then $\alpha$ and $\beta$ are constrained so as to produce a maximum possible prediction of $\cong .54$ for $P(i, 3)$. These constraints result from the fact that $\alpha$ and $\beta$ must both be in the range $(0,1)$. We made a table of the admissible values of $\alpha$ and $\beta$ which would satisfy Eq. 7 for $P(e, 3)=.67$. These sets of values were then substituted into Eq. 8 , with $x=0$, which implies a single channel. We found that $P(i, 3)$ was greatest when $\alpha=1, \beta=.375$; for these values, $P(i, 3)=$ .54. That is, a single-channel model predicts an advantage for sequential no less than .13 under the conditions of the present experiment. A single-channel magnitude model predicts an even larger difference. Since the maximum observed advantage for sequential for any $\mathrm{O}$ was only .03 and since the standard deviation of the difference for any $\mathrm{O}$ was only .02 , it is clear that a single-channel model may be ruled out of consideration.

Having ruled out a single-channel model, we next propose to examine attenuation models. For both the criterion and magnitude models, the degree of attention approaches 0 as $x$ approaches 1 . We propose, therefore, to place confidence limits on $x$ based on confidence limits for the observed difference. In the main experiment, the overall difference was .005 based on 4,452 observations for each condition. The standard deviation of the difference is

$$
\left[\frac{2(.67)(.33)}{4452}\right]^{1 / 2}=.01 \text {. }
$$

Thus, the 3-standard-deviation confidence limit for the difference, .03 , plus the observed difference, .005 , is .035 , or $.653-.688$. We then pick values for $\alpha, \beta$, and $\mathrm{x}$ which will satisfy Eqs. 7 and 8 for these limits, and which will also minimize the value of $x$. That is, we set $\mathrm{P}(\mathrm{e}, 3)=.688$ (Eq. 7) and $\mathrm{P}(\mathrm{i}, 3)=.653$ (Eq. 8). These equations have three unknowns $(\alpha, \beta$, and $\mathrm{x})$, so a variety of possible combinations of values can satisfy them. We made a table of possible values of $\alpha$ and $\beta$ satisfying Eq. 7, and for each of these found the value of $x$ satisfying Eq. 8. We found that $x$ took on its minimal value when $\alpha=1, \beta=.35$. For these values of $\alpha$ and $\beta$. $x$ $=.52$. Since this value of $\mathrm{x}$ was derived from the 3-standard-deviation confidence limit for the data. the probability is greater than .998 that $x>.52$. This bound of course is a minimum and can be raised by strengthening various assumptions. 
In particular, the bounds on $\mathrm{x}$ are more restricted for a model assuming that magnitude information is used in decisions. The magnitude model is described in Eqs. 11-14. Using the 3- $\sigma$ bounds of $.653-.688$ again. the calculations are straightforward. From Eq. 12 and the upper bound of .688 , we get: $.688=C\left(d^{\prime} .3\right)$. which gives $d^{\prime}=1.21$ (from tables in Swets. 1964). Then. from Eq. $13.653=1 / 3(.688)+2 / 3 \mathrm{C}\left(\mathrm{xd}^{\prime}, \mathrm{n}\right)$. Hence. $.636=$ $\mathrm{C}\left(\mathrm{xd}^{\prime}, 3\right)$; so $\mathrm{xd}^{\prime}=1.02$ and $\mathrm{x}=.84$. Thus, for the magnitude model, the probability is at least .998 that $x>.84$.

These bounds on $\mathrm{x}$ are model dependent, but they do place limits on the amount of attention that is possible, since our two example models were chosen to bracket the range of reasonable models. We are by no means arguing that these values of $x$ are, in fact, likely. The most parsimonious interpretation of the data holds that $x=1$, i.e., that there is no attention operating whatever. The burden of proof should now shift to those believing attention is operating on a limited-capacity input system. The direct implication of our results should be limited to the statement that capacity limitations and attention do not operate during relatively simple tactile detection tasks. Shiffrin and Gardner (1972) examined a more complex visual paradigm in which multiple signals were presented and failed to find attentional effects. However, the present study in the tactile domain leaves open the question of whether attentional effects might be seen if multiple signals were presented and/or multiple decisions were to be made.

\section{SUMMARY AND CONCLUSIONS}

The experiments compared simultaneous vs successive presentation of intervals, one of which contained a vibrotactile signal. Despite the fact that "attention" needed to be shared among the possible loci of stimulation during the simultaneous conditions, and was always directed toward the relevant signal site during sequential presentation, performance was identical in the two conditions. It was concluded that only minimal attentional effects could have been present. In order to validate this conclusion and to set bounds on the degree of attention that was possible, two quantitative models were considered. These "criterion" and "magnitude" models were chosen because they tended, respectively, to minimize and maximize the expected advantage for the sequential condition when attention was operating. It was found that single-channel models could definitely be ruled out of consideration and that, at most, only a small degree of attention could have been present. The most parsimonious interpretation of the results would hold that no attention is operating in the detection of near-threshold vibrotactile stimuli.

\section{REFERENCES}

Broadbent, D. E. Perception and communication. London: Pergamon, 1958.

Chase, W. G. Parameters of visual and memory search. Unpublished doctoral dissertation. University of Wisconsin, 1969.

Craig, J. C. Vibrotactile spatial summation. Perception \& Psychophysics, 1968, 4, 351-354.

Deutsch, J.. \& Deutsch, D. Attention: Some theoretical considerations. Psychological Review, 1963, 70, 80-90.

Deutsch. J., Deutsch. D., \& Lindsay, P. Comments on 'Selective attention: Stimulus or response.' Quarterly Journal of Experimental Psychology, 1967, 19. 362-368.

Estes, W. K., \& Taylor, H. A. A detection method and probabilistic models for assessing information processing from brief visual displays. Proceedings of the National Academy of Sciences of the United States of America, 1964, 52, 446-454.

Estes, W. K., \& Taylor, H. A. Visual detection in relation to display size and redundancy of critical elements. Perception \& Psychophysics, 1966, 1, 9-16.

Franzen, O., Markowitz, J., \& Swets, J. A. Spatially-limited attention to vibrotactile stimulation. Perception \& Psychophysics, 1970, 7, 193-196.

Guth, S. L. Letter to the editor on probability summation. Vision Research, 1971, 11, 747-750.

Moray, N. Attention: Selective processes in vision and hearing. New York: Academic Press, 1970.

$\mathrm{Ne}$ isser, U. Cognitive psychology. New York: Appleton-Century Crofts, 1967.

Norman, D. A., \& Rumelhart. D. E. A system for perception and memory. In D. A. Norman (Ed.), Models of human memory. New York: Academic Press, 1970.

Rumelhart, D. E. A multicomponent theory of the perception of briefly exposed visual displays. Journal of Mathematical Psychology, 1970, 7, 191-218.

Shiffrin, R. M., \& Gardner, G. T. Visual processing capacity and attentional control. Journal of Experimental Psychology, $1972,93,72-82$.

Shiffrin, R. M., Gardner, G. T., \& Allmeyer, D. H. On the degree of attention and capacity limitation in visual processing. Indiana Mathematical Psychology Report, No. 72-11. Indiana University, Bloomington, 1972.

Shiffrin, R. M., \& Gejsler, W. S. Visual recognition in a theory of information processing. Indiana Mathematical Psychology Report No. 72-10, Indiana University, Bloomington, 1972.

Swets, J. A. (Ed.) Signal detection and recognition by human observers. New York: Wiley, 1964.

Treisman, A. Strategies and models of selective attention. Psychological Review, 1969, 76, 282-299.

(Received for publication September 21, 1972; revision received November 20,1972.) 